\title{
New developments in the field of high current ECR ion sources at the IAP RAS
}

\author{
V.A. Skalyga, S.V. Golubev, I.V. Izotov, M.Yu. Kazakov, R.L. Lapin, S.V. Razin, \\ A.V. Sidorov, R.A. Shaposhnikov, A.F. Bokhanov
}

Institute of Applied Physics of Russian academy of Sciences, Nizhny Novgorod, Russia, skalyga@ipfran.ru

Investigations of pulsed ECR discharge in an open magnetic trap under conditions of powerful ECR heating with gyrotron $\mathrm{mm}$-waveband radiation were carried out over the last 20 years at the Institute of Applied Physics (IAP RAS, Nizhniy Novgorod, Russia) [1-5]. In the beginning the work was devoted to development of a high frequency ECR source of multi-charged ions with outstanding parameters of plasma heating $(37.5 \mathrm{GHz}, 100 \mathrm{~kW})$. According to Geller's scaling laws [6] such increase in frequency and power in comparison to conventional ECRIS was expected to boost the ion source performance and provide a significant progress in ECRIS development. However, due to short pulse operation mode and low repetition rate of the used gyrotrons (pulse duration $<1 \mathrm{~ms}, 0.1$ $\mathrm{Hz}$ ) breakdown and discharge conditions similar to a conventional ECRIS were unreachable. The minimum neutral gas pressure was two orders higher $\left(10^{-4} \mathrm{mbar}\right)$ and the plasma parameters differed significantly from conventional ECRIS. After years, this work resulted in development of a new type of ion source - high current gasdynamic ion source.

Experimental studies of gasdynamic ECRIS were performed at SMIS 37 facility, schematically depicted in Fig. 1. The plasma was created by 37.5 and $75 \mathrm{GHz}$ gyrotron radiation with power up to $100 \mathrm{~kW}$. Gasdynamic ECRIS has demonstrated a good performance producing high current (100-300 mA) multi-charged ion beams with moderate average charge ( $Z=4-5$ for argon). Gasdynamic ECRIS has appeared to be especially effective in low emittance hydrogen and deuterium beams formation. Proton beams with current up to $500 \mathrm{emA}$, RMS emittance below $0.07 \pi \cdot \mathrm{mm} \cdot \mathrm{mrad}$ have been demonstrated in recent experiments.

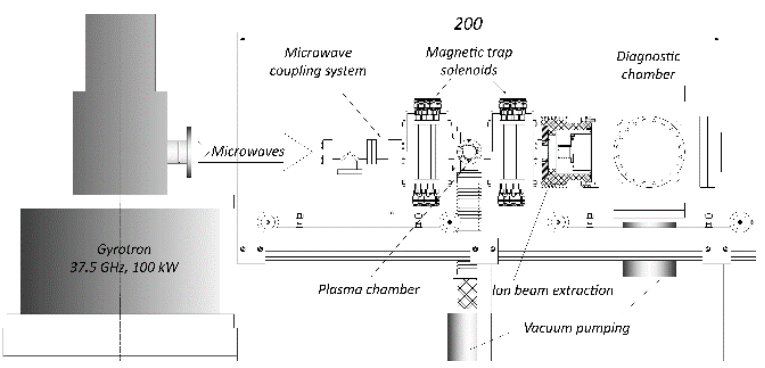

Fig. 1. SMIS 37 experimental facility.

Experiments aimed to demonstrate the benefits of the high current gasdynamic ECR ion source as a part of D-D neutron generator were

Measurement of the produced neutron flux was performed by bombarding a heavy ice target with 300
emA D ${ }^{+}$beam accelerated to $45 \mathrm{keV}$ energy. The $\mathrm{D}_{2} \mathrm{O}$ target yield was $10^{9} \mathrm{~s}^{-1}$. It is worth noting that the area of the target was about $1 \mathrm{~cm}^{2}$, which corresponds to a neutron flux density of about $10^{9} \mathrm{~cm}^{-2} \cdot \mathrm{s}^{-1}$ at the target.

As it was demonstrated a high-current ECR ion source with quasi-gasdynamic plasma confinement and heating with gyrotron microwave radiation allows the formation of light ion beams with uniquely low emittance (for a given current level). Such a low emittance enables focusing of the ion beam into a small spot. So it could be used as alternative approach for creation of a point-like neutron source for neutronography. A magnetic lens was used for beam focusing as the next step. Measurements have shown that lens focus for $1.3 \mathrm{~T}$ field was situated approximately in 10 $\mathrm{cm}$ from lens center. Example of scintillator luminescence image induced by $80 \mathrm{keV}, 60 \mathrm{~mA}$ deuterium beam is presented in fig. 2. The best result for focused beam full width at half maximum was $0.75 \mathrm{~mm}$.

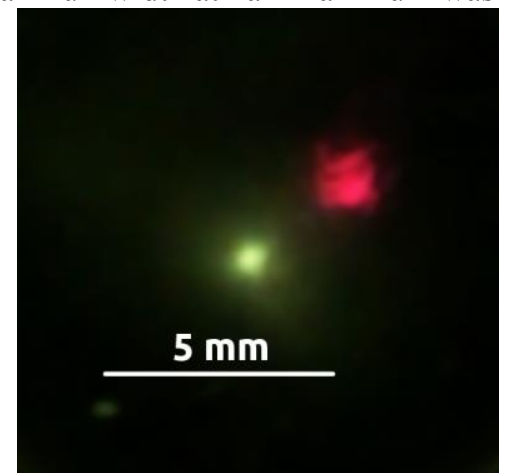

Fig. 2. Photograph of scintillator luminescence (green glow) induced by $80 \mathrm{~mA}$ hydrogen beam in magnetic lens focal plane (red glow corresponds to optical emission from plasma through the plasma electrode aperture)

Obtained focused ion beam was used for $\mathrm{TiD}_{2}$ target bombardment. As a result the "point-like" neutron emission with total yield about $10^{10} \mathrm{~s}^{-1}$ have been demonstrated.

Another direction for experimental studies is $\mathrm{H}^{-}$ ion source development. $\mathrm{H}^{-}$ion sources are of great demand for charge exchange injection into cyclotrons and storage rings. It has been recently demonstrated that a gasdynamic ECR ion source based on ECR discharge in a simple mirror trap is very efficient for proton beam production $[7,8]$. Therefore, it was suggested to use the gasdynamic plasma source as the first stage of an $\mathrm{H}^{-}$ion source based on volumetric production through dissociative electron attachment $[9,10]$ (DEA). 
The first experiments were performed in a pulsed mode with $37 \mathrm{GHz}$ / up to $100 \mathrm{~kW}$ gyrotron radiation in a dual-trap magnetic system, consisting of two identical simple mirror traps. The schematic of the experimental facility is presented in fig.3. The first trap was used for plasma production under ECR conditions. Dense hydrogen plasma flux (with estimated current density of about 1-5 A/ $\mathrm{cm}^{2}$ ) from the first trap was allowed to flow into the second trap through a perforated conducting plate. The grid placed between the traps prevents microwave leakage into the second part to avoid electron heating there. Such configuration was chosen to produce a flux of "hot" electrons (energy $50-100 \mathrm{eV}$ ), which can effectively ionize and excite high vibrational states of hydrogen molecules in the second trap, and to produce anions there as the result of DEA with "cold" electrons (energy < $10 \mathrm{eV}$ ). Thus, the plasma confined in the second trap presumably consists of two electron populations enabling the volume production of $\mathrm{H}^{-}$ions. Such approach is similar to the one suggested in [11] where heating $2.45 \mathrm{GHz}$ ECR discharge was used as a plasma cathode producing "hot" electrons.

In case of $1 \mathrm{~mm}$ diameter of the hole for beam extraction negative ion current was up to $0,7 \mathrm{~mA}$. The corresponding ion current density was $80 \mathrm{~mA} / \mathrm{cm}^{2}$. Such results makes further investigations very perspective.

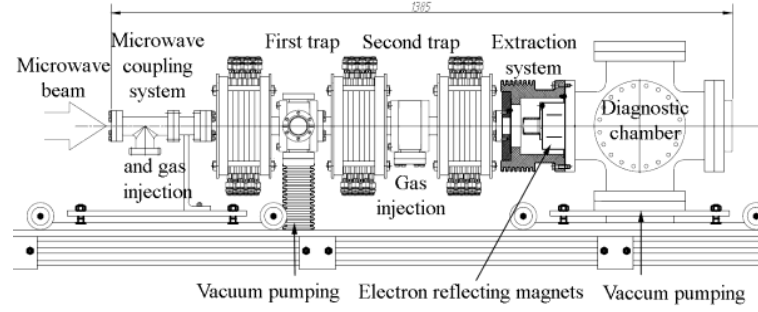

Fig. 3. Scheme of the experimental facility. Typical negative ion current waveform. Microwave power 80 $\mathrm{kW}$, constant gas flow into the first trap and pulsed injection into the second one.

The main part of previous experiments were carried out in a pulsed operation mode. Preliminary studies of plasma parameters were performed using a CW source with $24 \mathrm{GHz} / 5 \mathrm{~kW}$ gyrotron heating. Obtained experimental results have demonstrated that all gasdynamic source advantages could be realized in $\mathrm{CW}$ operation. To continue development of a $\mathrm{CW}$ gasdynamic ion source a new experimental facility named GISMO (Gasdynamic Ion Source for Multipurpose Operation) is under construction at the IAP RAS. Future facility have been named GISMO (Gasdynamic Ion Source for Multipurpose Operation). This facility is aimed to produce continuous high-current $(>200 \mathrm{~mA})$ ion beams with low emittance $(<0.2 \pi \cdot \mathrm{mm} \cdot \mathrm{mrad})$. The scheme of the future experimental facility is show in figure 1 . The key elements of the setup are $28 \mathrm{GHz} / 10 \mathrm{~kW}$ and 37,5 $\mathrm{GHz} / 20 \mathrm{~kW} \mathrm{CW}$ gyrotrons manufactured by Gycom [12]. This microwave generators will be equipped with power supplies suitable for $\mathrm{CW}$ or pulsed opera- tion. A fully permanent magnet magnetic trap will be used for plasma confinement. Magnetic field configuration was designed to be similar to a simple mirror trap close to the system axis with field strength at magnetic mirrors of $1.5 \mathrm{~T}$ and mirror ratio close to 6 . Distance between magnetic mirrors is about $12 \mathrm{~cm}$. For ion beam extraction it is planed to use 3 or 4electrode system with maximum acceleration voltage up to $100 \mathrm{kV}$. Such extraction requires development of an appropriate high-voltage insulation of the discharge chamber from other parts. In this regard, one of the key elements of the installation is the DC-break of the microwave transmission line. It was proposed to be implement a quasioptical system shown in Fig. 1. Plasma chamber will be $30 \mathrm{~cm}$ in length and $4 \mathrm{~cm}$ in diameter. It is equipped with water cooling along whole surface from the coupling system to the flange.

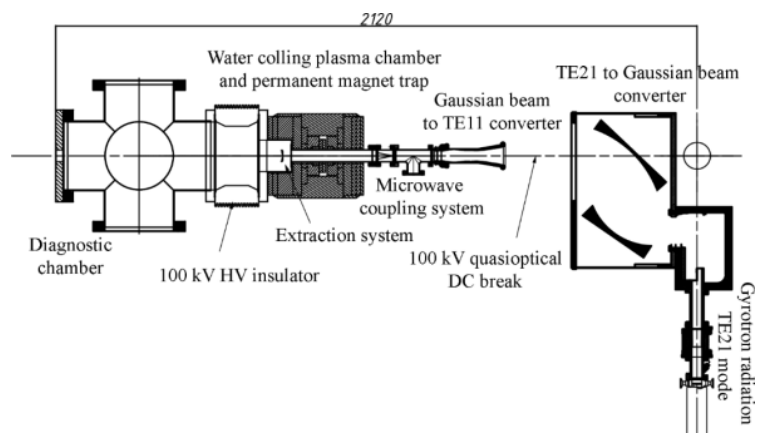

Fig. 4. Scheme of the GISMO 28/37 CW high current ion source.

The work is being realized in frames of realization of Federal targeted program R\&D in Priority Fields of the S\&T Complex of Russia (2014-2020) contract \#14.604.21.0195 (unique identification number RFMEFI60417X0195).

\section{References}

1. S.V. Golubev et al., Rev Sci. Instrum. 75, 1675 (2004).

2. S.V. Golubev et al., Trans. Fus. Sci. and Tech. 47, 345 (2005). (2006).

3. A. Sidorov et al., Rev Sci. Instrum. 77, 03A341-1

4. V. Skalyga et al., Plasma Sources Sci. Tech. 15, 727 (2006).

5. S. Golubev et al., Nucl. Instrum. Meth. in Phys. Research B 256, 537 (2007).

6. R. Geller, Electron cyclotron resonance ion sources and ECR plasmas (Institute of Physics, Bristol, 1996).

7. V.A. Skalyga et al., Rev. Sci. Instrum. 85, 02A702-13 (2014).

8. V. Skalyga et al., Rev. Sci. Instrum. 87, $02 \mathrm{~A} 716$ (2016)

9. M. Bacal, G.W. Hamilton, Phys. Rev. Lett. 42, 1538 (1979).

10. M. Bacal Nuclear Fusion 79, 02 A516 (2008).

11. R. Keller et al., in AIP Conf. Proceedings 773 (American Inst. Of Physics, Melville, NY. 2005), p. 104 106.

12. www.gycom.ru 